\title{
AN IMPROVED LIGHT DARK BOX TEST BY USING A REAL-TIME VIDEO TRACKING SYSTEM
}

\author{
Sareesh Naduvil Narayanan ${ }^{1}$ * and Raju Suresh KumaR ${ }^{2}$ \\ Department of Physiology, Melaka Manipal Medical College (Manipal Campus), \\ Manipal University, Manipal 576104, India
}

(Received: April 4, 2018; accepted: August 6, 2018)

\begin{abstract}
In the behavioral science field, many of the oldest tests have still most frequently been used almost in the same way for decades. The subjective influence of human observer and the large inter-observer and interlab differences are substantial among these tests. This necessitates the possibility of using technological innovations for behavioral science to obtain new parameters, results and insights as well. The light-dark box (LDB) test is a characteristic tool used to assess anxiety in rodents. A complete behavioral analysis (including both anxiety and locomotion parameters) is not possible by performing traditional LDB test protocol, as it lacks the usage of a real-time video recording of the test. In the current report, we describe an improved approach to conduct LDB test using a real-time video tracking system.
\end{abstract}

Keywords: Light-dark box - emotion - anxiety - locomotion - behavior

\section{INTRODUCTION}

Anxiety is a generalized response to an unknown threat or internal conflict [12, 47]. Since the fundamental aspect of anxiety appears to be uncertainty, it has been recommended that anxiety can only be understood by considering some of its cognitive aspects [3]. The pursuit for tools to measure anxiety has a long history. In order to evaluate anxiety behaviors in rodents, researchers have designed several approaches such as light-dark box (LDB) test [3, 6, 13] elevated plus maze (EPM) test [35] and open field test $[18,25]$. These tests have been widely used in preclinical animal studies even today $[21,22,32,43]$. The LDB test is a characteristic tool used to assess anxiety in rodents and it was developed by Crawly and colleagues [13]. LDB test has been originally developed to evaluate the anxiety in mice $[13,14,15,32]$ and later found to be useful in assessing anxiety in rats $[7,8]$. LDB test is an excellent tool

* Present addresses: ${ }^{1}$ Department of Physiology, RAK College of Medical Sciences, RAK Medical \& Health Sciences University, PO Box. 11172, Ras Al Khaimah, UAE. ${ }^{2}$ College of Science and Health Professions - Jeddah, King Saud Bin Abdulaziz University for Health Sciences, National Guard Health Affairs, P. O. Box 9515, Jeddah 21423, Kingdom of Saudi Arabia.

Corresponding author; e-mail address: sareeshnn@yahoo.co.in 
in evaluating the effectiveness of anxiolytic [50] and anxiogenic agents in rodents $[30,41]$.

The LDB apparatus consists of a brightly illuminated chamber and a dark chamber with an opening which connects the two chambers [3]. Researchers have attempted various approaches for acquiring data during LDB test. Chen et al. [9] have used computer-controlled $4 \times 16$ array of photo beams to record the time spent in each chamber of LDB automatically. On the other hand, Bedse et al. [4] performed LDB test by using activity Monitor v5.10 (MED Associates) which detects beam breaks (from 16 infrared beams) to determine position of rats during LDB test. In another study, Diaz et al. [19] videotaped the activity of rats in the light area during LDB test and these were used to score anxiety related parameters by an experimenter manually. Sarwar et al. [42] has conducted the test by videotaping the rat behavior in the apparatus, but rat's activity in the dark compartment has not been reported. Although these reports demonstrated the use of technology for conducting LBD test, in some of the very recent reports it can be observed that this test has been performed entirely manually $[17,21,39,44]$. These methodological variations make it difficult to replicate or compare the results different laboratories. Moreover, there are several other parameters that convey behavioral change, possibly due to a drug treatment or stressor could not be recorded if the test is conducted manually; without a real-time video tracking system that records activities of rats in both the compartments. Some of these include distance travelled, velocity of animal, parallelism index, other specific behaviors such as grooming, rearing, freezing in both the chambers. Additionally, even by using beam breakers some of these parameters are difficult to record. The current study was designed in this context and it was an attempt to particularly evaluate the following aspects compared to previous studies conducted in this area.

a) Possibility of conducting LDB test in a room illuminated with only 5 lux or less. If this can be done, one needs not to close the dark chamber by a lid and animal behavior or movements can be video-tracked from above in both the chambers. This will enable us to critically evaluate anxiety and locomotion patterns in animals during the test. Although reports are available on the utility of LDB test for assessing anxiogenic [30,41] and anxiolytic [50] behaviors in animals, its potential use in exploring locomotion related variables is rarely reported. Most of the time, researchers have to depend on other behavioral assays $[18,25]$ to evaluate locomotor behavior even after conducting LDB test. One can overcome this hurdle if LDB test is done with an automatic real-time video tracking system that records the activates of rats in both the chambers.

b) Possibility of acquiring LDB test data entirely by a computerized automaticdata acquisition system and to obtain the animal path trace for further evidence of animal activity in the apparatus: There are only a very few reports mentioning the use of real-time video tracking of animal activity in the LDB. In the majority of the reports, video recording was done only in the light chamber $[19,24]$ and the path taken by each rat in LDB was rarely reported. In addition, computerized automatic data acquisition was also not found to be used in most of the recent reports [21, 39, 
44]. The subjective influence of human observer is a major confounding factor in these type of behavioral assays and so the use of technological assistance (automated systems) is heeded which would solve the reliability issues to some extent $[1,46]$.

c) The possibility of using the commercially available PanLab software for LDB test. This software has been used by several researchers for acquiring data in various behavioral tests, such as open field test [29], elevated plus maze test [37], and Morris water maze test $[31,36]$. However, it's potential utility in assisting LDB test has not yet been explored. The current study is the first of its kind to use this software in performing LDB test.

d) The possibility of obtaining novel, important anxiety and locomotion related parameters by performing minor reforms on test procedure/apparatus size. There is no consensus in the literature regarding the initial release of animal into light/dark chamber $[23,28]$. In some studies, it has been reported that the rat was placed initially into the light chamber [2,44] and in some others into the dark first [17, 22]. Placing rat first in the dark chamber will enable the experimenter to record latency to enter the light, and this can be used as a measure of anxiety. The possibility of obtaining this parameter (latency to enter to light) was also tested in the current study. Additionally, LDB apparatus size variations are evident in some of the reports [10, $23,28]$. Both the chambers of the apparatus were made equal and its effectiveness in obtaining distance travelled in each chamber was also studied.

A consensus is not provided in reports regarding the total duration of LDB experiment $[5,16,45]$. Some researchers have conducted the test for 5 min duration $[5,11$, $23]$ and several others have conducted it for 10 min duration [2, 4, 22]. Variations are evident in many of the reports and majority of the reports suggest 5 min duration is appropriate for the test $[5,11,23]$. Conventionally, the number of entries into each compartment (specifically light chamber) and time spent there are measured [15]. Entries to the bright chamber and the time spent there are generally used as indices of anxiety in mice [10]. Additionally, parameters such as latency time in dark chamber [49] and rears in the bright $[5,45]$ were also measured and reported by different researchers. Keeping in mind some of the aforementioned limitations of traditional LDB test procedure and to improve data acquisition during this test, we describe an improved LDB test procedure by using a real-time video tracking system.

\section{MATERIALS AND METHODS}

\section{Animals, maintenance and experimental groups}

Thirty-six male albino Wistar rats (6-8 weeks old) were obtained from the Central Animal Research Facility (CARF). Rats were housed in polypropylene cages measuring $41 \mathrm{~cm} \times 28 \mathrm{~cm} \times 14 \mathrm{~cm}$. Three rats were housed in each polypropylene cage. All animals were maintained in 12:12 hr L:D environment, in an air-conditioned 
room in the central animal house and were fed with water and food ad libitum. Care was taken to handle the rats in humane manner and all precautions were taken to use only minimum required number of animals to generate significant data. The Institutional Animal Ethics Committee (IAEC) has approved all procedures used in the study. To avoid observer's bias during the experiment, an independent person coded animals before subjecting them to various experiments. All animals were screened using EPM test for evaluating anxiety levels. Those animals spent significantly less time on the open arms were categorized into anxiety group. After the EPM test five rats were found to be displaying anxiety-like behavior and were designated as test group. Four from the remaining were randomly selected and used for standardizing the modified video tracking method. Another five control rats were tested on LDB and their data was compared with that of anxiety group data to validate the new approach.

\section{Elevated plus maze test}

The elevated plus maze (EPM) apparatus is a plus shaped maze, made out of black Plexiglass. It was indigenously made as per standard measurements to suit our needs. It has two closed $(51 \mathrm{~cm} \times 10 \mathrm{~cm} \times 40 \mathrm{~cm})$ and two open arms $(51 \mathrm{~cm} \times 10 \mathrm{~cm})$ opposite to each other. The open and closed arms are connected by a central platform measuring $10 \mathrm{~cm} \times 10 \mathrm{~cm}$. The apparatus was elevated to a height of $52 \mathrm{~cm}$ from the floor. The test and data acquisition were performed by following an earlier published report [37].

\section{Light-dark box (LDB), camera and lighting adjustments}

The apparatus was made indigenously to suit our improved LDB test (Fig. 1A). The light and dark boxes are made out of white and black Plexiglass, respectively. Although apparatus size variations are evident across reports, in the current study the size of both the boxes were made equal $(35 \mathrm{~cm} \times 35 \mathrm{~cm} \times 35 \mathrm{~cm})$. The two boxes are connected by an opening measuring $8 \mathrm{~cm} \times 8 \mathrm{~cm}$ (Fig. 1A). Right above the LDB, a video camera (Sony) was fixed on a tripod stand and it was kept immobile during the whole experiment (Fig. 1B). It was connected to a PC installed with Panlab SMART (version 2.5 image analysis system) software (Barcelona, Spain) for data acquisition. The LDB and PC was kept in different rooms to minimize the experimenter presence induced behavioral change in rat. The LDB test was conducted between 07:00 pm to 11:00 pm in an interior room (hermetic to exterior light as possible). The experimental room lighting was controlled to obtain 5 lux in the dark box and illumination in the light box is adjusted to obtain 600 lux by a $60 \mathrm{~W}$ incandescent light bulb. 


\section{Preparation of Panlab SMART Image analysis system for the modified test}

The lighting and contrast camera settings were configured in the SMART software as required. Briefly, in the camera settings the frame position was selected and fixed. In order to calibrate the area of LDB, the horizontal and vertical calibration options were selected and calibrated. To have a record of various behaviors (eg; rearing, grooming) during the experiment, event marker is used. Event marker setting was opened in the software and computer key board buttons 0-9 were assigned for various behaviors visualized by the experimenter. The name of the behaviors to note was entered in a case selected by the experimenter. These details (the file path) were saved in appropriate destination folders in the PC. Following this, various zones were drawn after opening zone editor dialogue box. To represent light and dark zones in the current study (light and dark) the SMART specific drawn tools were used. Red and blue color rectangles were used to depict dark and light box respectively (Fig. 1C). A special zone, named exclusion zone (outside both the zones) has been conceived to save resources, and prevent interferences of changes that may occur around the experimental field (i.e. Light/Dark box). Any image from this zone, was not processed by the SMART system. The part of the image that does not correspond to one of the previously defined zone is recognized as exclusion zone (Fig. 1C). The definition of zones and all their properties were saved as a zone file in the PC. After this the zone editor dialogue box was closed to return to the main screen.

\section{Performing the experiment using SMART software}

In the data acquisition single-subject tracking (SST) was selected. After this, a tracking file was created and later, a zone file which was prepared earlier was opened. In SMART a standard Windows ${ }^{\circledR}$ dialogue box will open enabling the selected file to be opened. After this the subject list was created. The subject list enable the experimenter to have a list of all the animals used in the experiment saved, as well as the order in which they have been used and this can be referred again later. The duration of the experiment is entered in the timing menu. A wait time (latency) to be set prior to as well as the total duration (acquisition) of the experiment is set here. In the current study the acquisition duration was set as 5 minutes. For automatic recording, following setting up the time, the subject detection technique for tracking has to be done. But in the current study, manual tracking option was selected and therefore this was not adjusted. Following this a photo of the scenery without the animal was taken. This snapshot of the scenery is needed by the software to detect the mobile parts of the new images it will get during data acquisition (by subtraction). Finally, after configuring the movement threshold (resting, slow and fast) the experiment was started. 

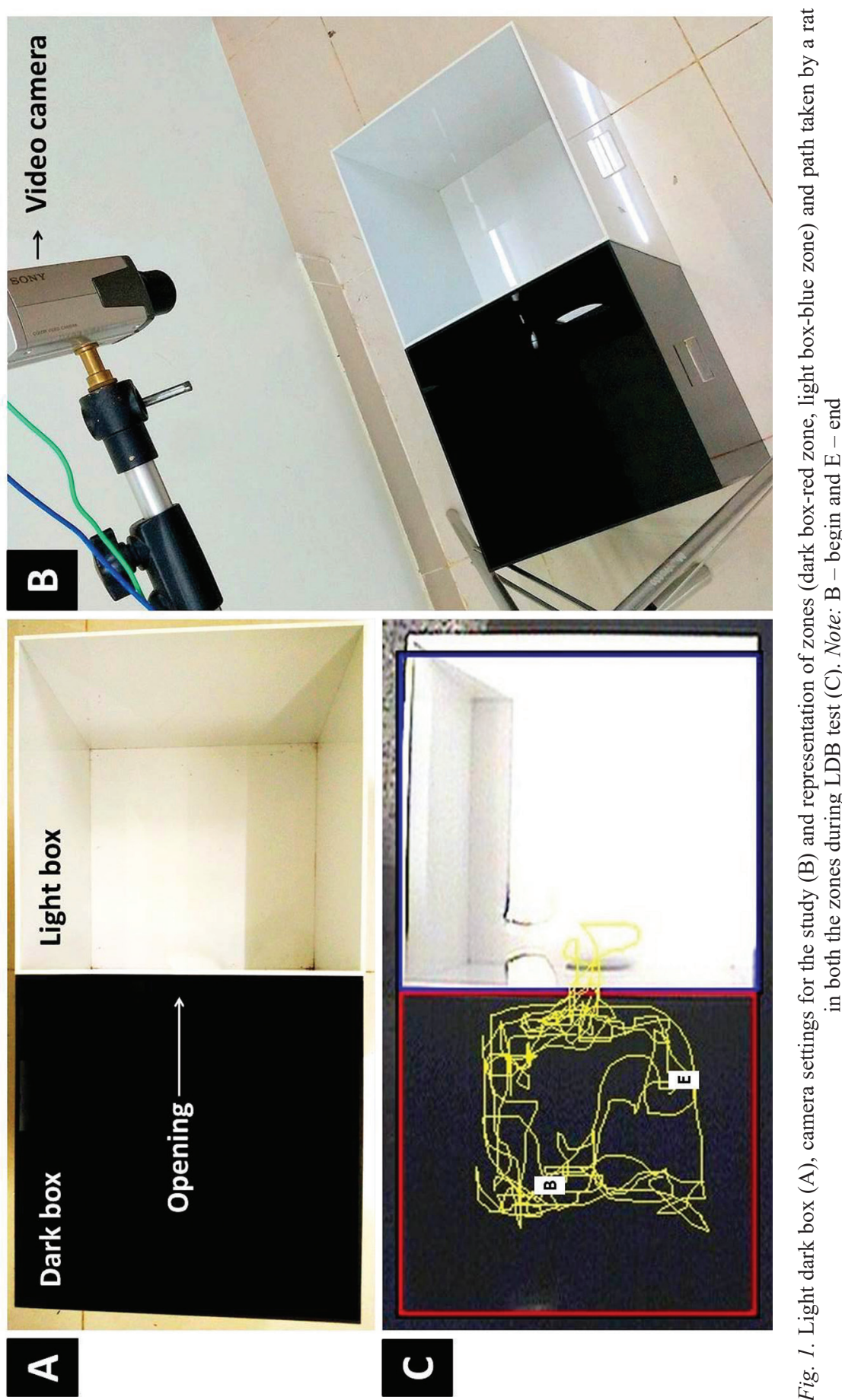

Acta Biologica Hungarica 69, 2018 
At the start of the test, each rat was placed in the dark box and start icon was pressed immediately on the software. Soon after this, the experimenter moved to another room to minimize the experimenter induced behavioral change of the rat which would otherwise be a confounding factor. A single test with 5 min duration was applied to each rat and during this period each rat's behavior was thoroughly analyzed and recorded. A trained individual tracked the rat path (in real-time) during the test. Percentage of time spent in light and dark boxes, latency to emerge to light box, number of entries to light $\&$ dark boxes, distance travelled in light and dark boxes, and total distance travelled were recorded and analyzed. In addition, during real-time data acquisition, by pressing the corresponding numerical key on the keyboard, number of rearing event was also recorded. Soon after each rat was tested, the whole apparatus was cleaned using $0.1 \%$ acetic acid solution.

\section{Validation of the improved method}

When designing new experimental methodologies or approaches, it is pivotal to consider their reliability and validity. The LDB test is considered as a reliable and valid method to evaluate the anxiety-like behavior in rodents. Similarly, it is certain that SMART offers a dependable data when used appropriately. When these are used together as in the current modified LDB test, whether it measured what it claims to (such as anxiety-like behavior), was tested by doing a comparison of data obtained from different groups. Moreover, this comparison helps to give an idea about the utility of the improved method in obtaining other novel parameters (locomotion) and this possibly will substantiate the utility of the improved technique.

\section{Statistical analysis}

The descriptive statistics of various outcome measures were represented as mean $\pm \mathrm{SE}$. Significant difference between groups were analyzed by Student's ' $\mathrm{t}$ '-test. $p<0.05$ was considered as statistically significant at $5 \%$ level of significance. GraphPad Prism software (version 5.01; CA, USA) was used for data analysis.

\section{RESULTS}

Comparison of the data of the various groups' was done as an attempt to examine the validity of the modified experimental setup and approach. It is apparent from the results that the percentage time spent in light compartment and distance travelled in the same was decreased in test group animals (Fig. 2A, B; control vs test, Student's $t$-test, $\left.{ }^{*} p<0.05 ; * * * p<0.001\right)$ compared to control group, indicating anxiety, like behavior in these rats. Rearing frequency positively correlates with the exploratory behavior in rats, and in the current study test group rats performed significantly less 
rearing events compared to the control group (Fig. 2D; control vs test, Student's $t$-test, $* p<0.01)$. These results demonstrate that the current modified LDB test is sensitive to anxiety, like behavior in rats and it measures what it was supposed to measure. The anxiety group animals did visit the light-box several times and it was not significantly different compared to controls (Fig. 2E). The latency to reach light-box was also not found to be different in test group compared to control animals (Fig. 2F). Real time videotaped records suggest more anxious nature of test group as depicted by their trajectories (Fig. 3B, $\mathrm{B}_{1}$ ) compared to controls (Fig. 3A, $\mathrm{A}_{1}$ ) in the light compartment
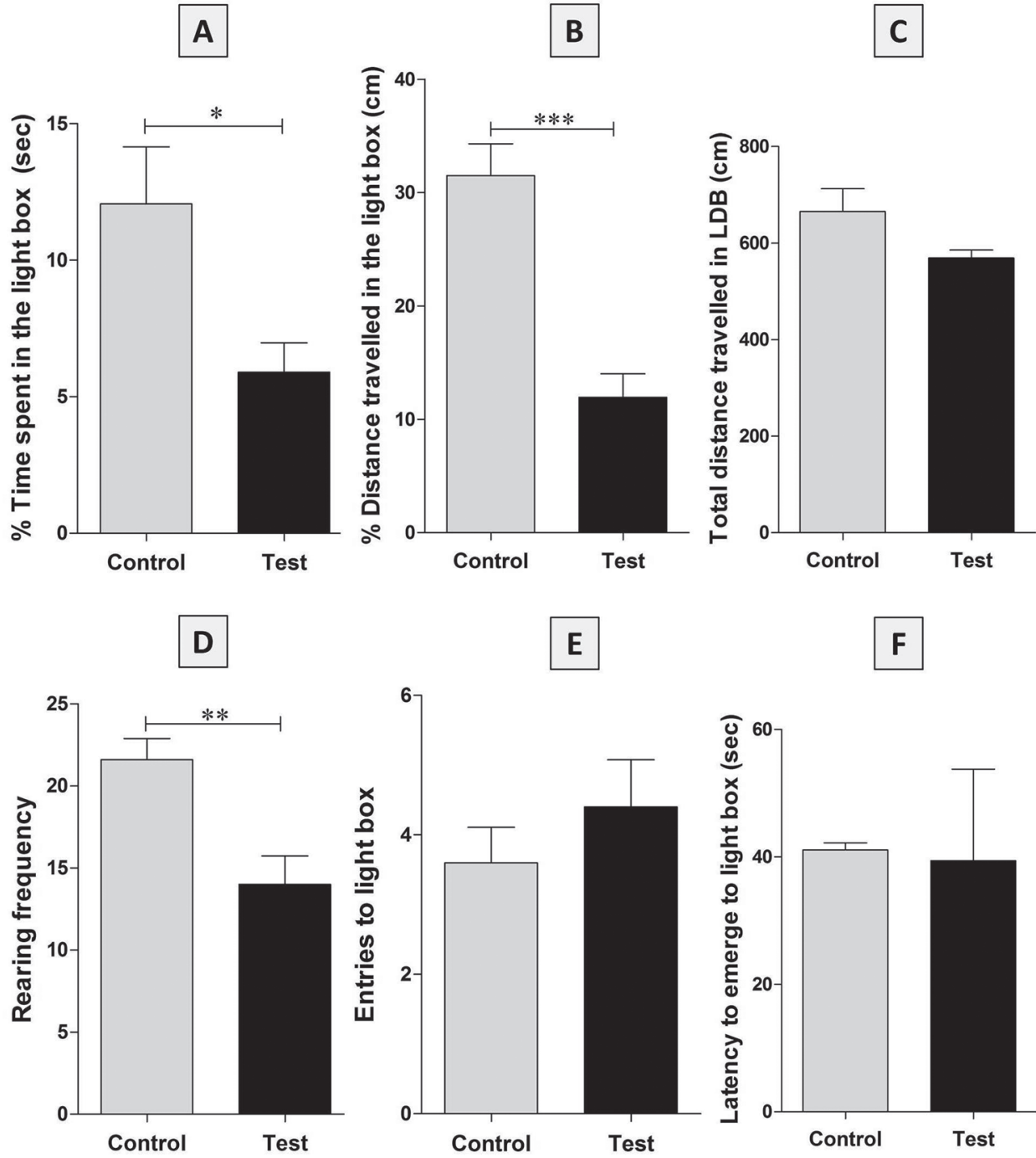

Fig. 2. Comparison of various parameters recorded during modified LDB test in control and test group animals. Note: Error bars represent standard errors (SE). $p^{*}<0.05, p^{* *}<0.01$ and $p^{* * *<0.001}$ 


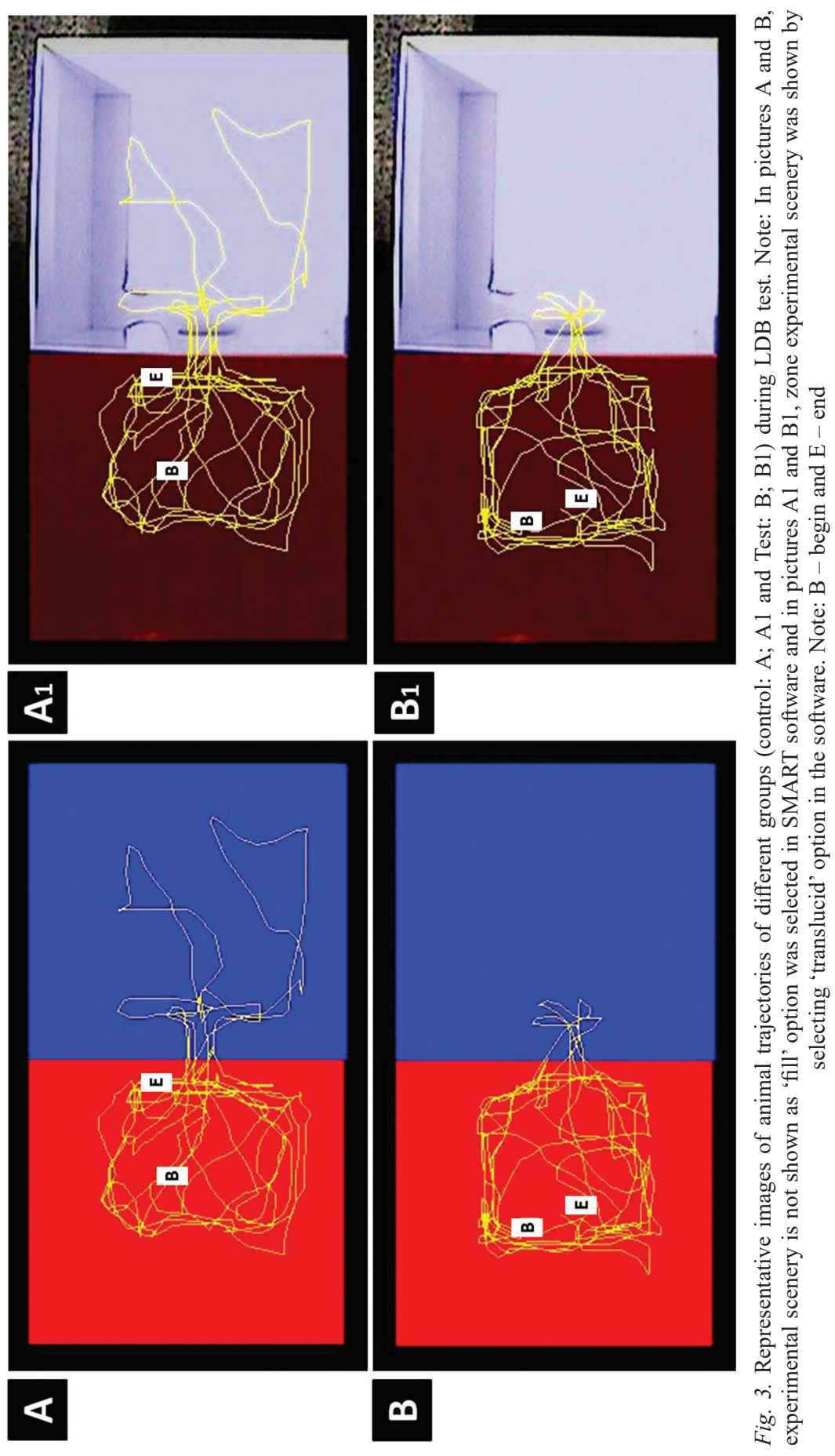

Acta Biologica Hungarica 69, 2018 
light-box to dark. Total distance travelled in the LDB is one of the locomotion related variable recorded in the study and it was not found to be significantly different between groups (Fig. 2C). The effectiveness of current approach is explicit, as demonstrated by significantly less time spent in the light-box and less distance travelled in the same by high anxiety group compared to control group.

\section{DISCUSSION}

In the behavioral science field, many older tests have still most frequently been used almost in the same way for decades [26, 27]. The subjective influence of human observer and the large inter-observer and inter-lab differences are substantial in these tests. This necessitates the possibility of using technological innovations for behavioral science to obtain new parameters, results and insights as well. Researchers have the opinion that automated methods could solve the reliability issues $[1,46]$. Since in an automated method a machine scores the same stream of events almost identically in every trial, to an extent the reproducibility issues could also be solved.

Frequently it is the tradition and the apparent need for simplicity which have restricted the validation and use of automated methods and technological innovation in behavioral science field. The advantages of automated methods, such as reliability, more precision, expeditious and possibility of triumphing new parameters, cannot be ignored. However, the necessity of appropriate validation of these methods and technological innovations should be considered. Several attempts have been made to improvise the LDB test [6]. Researchers have also tried to minimize the subjective influence of the human observer which would otherwise be a confounding factor. In the current study a typical LDB test was performed with modification. While performing the traditional LDB test, it is difficult to observe animal activity in the dark box as it is covered by a lid [34]. However, in the current modified method, this was kept open, but illumination in the experiment room was adjusted to that of a dark-box, as it was closed. This helped us in recording several aspects of animal behavior (in the dark box) which would otherwise could not. Additionally, distance travelled, trajectory of each animal (locomotion pattern) and other behaviors such as rearing, grooming, freezing, risk assessment behaviors, such as head dips and protected stretches could also be studied. Although in the current report, velocity of each animal was not reported, this can also be recorded and which would give an idea about effects of various treatments (drugs or stressors) on locomotion.

The traditional LDB test is typically developed to test anxiety behavior of rodents, therefore, locomotion related behaviors are rarely considered during this test. The requirement of a sophisticated tool (software) to measure locomotor behaviors during LDB test further hinders this task. Among reports it is evident that, while performing LDB test, light chamber parameters have been considered over the dark box parameters [6]. As a result, distance travelled in the dark chamber, total distance travelled in the apparatus, and other behaviors were not taken into consideration (in fact it remained unknown at least in some experiments) when this test was conducted 
manually $[17,21,39,44]$. Many a times, researchers have to perform additional tests to get an idea about animal locomotion even after conducting LDB test. It is seen that multiple behavioral assays in same group of rats leads to changes in animal behavior [47], sometimes conflicting outcomes and this exacerbates data interpretation [20]. Researchers have suggested the possibility of performing 'combined anxiety test' (EPM, LDB and OP) to overcome some of these difficulties [40]. In this context, the present study is very pertinent. A researcher will be able to get a thorough analysis of both anxiety and locomotor behavior of rodents while performing the present modified approach. Additionally, in some anxiety and depression models (social isolation and maternal separation stress) freezing behavior is evident [33] and when such model animals are kept in the dark box, during the entire duration of the experiment, it likely to be there. Unfortunately, in the traditional LDB test, no data can be obtained during such a situation except the time spent in the dark chamber.

Compared other studies $[11,28]$ to the current study, we used LDB with each of its compartment has same measurements. We believe that two chambers should have equal measurements if one wishes to compare the distance travelled in each chamber. As depicted in the results, in the current study we have compared the distance travelled by rats in light chamber and it is evident from the results that, anxious rats travelled less distance in the light chamber. This outcome further substantiates the sensitivity of the modified method.

Moreover, in this study, since the data acquisition was completely done by an automated system, the subjective influence of human observer was minimized significantly. It is evident from the results that the SMART software can effectively be used for LDB test. However, while performing the real-time video tracking of the rat activity, it was challenging for us to create the lighting condition of the experiment room as that of the dark-box when it was closed. By adjusting the illumination in the test room, we could overcome this challenge and this resulted in achieving better outcomes as seen from the results. Real-time video tracking of the path of the subject (in the light-dark box) did potentiate the behavior analysis, and so it provided further evidence of rat activity in the chambers. Subject path tracing has rarely been reported in other published reports. Although in the current study manual tracking mode was selected, it has been conducted in real-time, as the rat performed various activity in the apparatus. Hence, in reality it is a real-time video tracking. There is a provision in the software to perform automated tracking and this can be done by adjusting the light threshold in the software. It is accepted that a measure can be reliable and not valid, but a valid measure is always a reliable measure. Comparison of the data of various groups was done to test this uncertainty and it was clear from the data of the current report that the test in its modified way is sensitive to animal emotion such as anxiety. Sensitivity of this method to a drug effect is not being tested in the current study, and this can be one of the limitation of the study. Therefore a future study in this regard would help to further validate the method. Although the possibility of using commercially available software is described in this paper, other similar open source software can also be used for preforming the modified approach. 


\section{CONCLUSIONS}

A new approach to conduct LDB test using a real-time video tracking system was found to be effective in evaluating both anxiety and locomotion related behaviors of rats. The test in its modified way is sensitive to animal emotion such as anxiety and it helped to achieve a complete behavioral assay in rat anxiety model. The present approach can also be used for recording several other aspects of animal behavior which are not possible to be recorded and studied by performing traditional LDB test protocol.

\section{ACKNOWLEDGEMENTS}

Research funding from Indian Council of Medical Research (ICMR) to SNN (File No: 5/10/FR/21/2011RHN, IRIS ID: 2011-08800) is gratefully acknowledged.

\section{REFERENCES}

1. Aarts, E., Maroteaux, G., Loos, M., Koopmans, B., Kovačević, J., Smit, A. B., Verhage, M., Sluis, Sv. (2015) Neuro-BSIK Mouse Phenomics Consortium: The light spot test: Measuring anxiety in mice in an automated home-cage environment. Behav. Brain. Res. 294, 123-130.

2. Antsiferova, A., Kopaeva, M., Kashkarov, P. (2018) Effects of prolonged silver nanoparticle exposure on the contextual cognition and behavior of mammals. Materials (Basel). 11. pii: E558.

3. Aulich, D. (1976) Escape versus exploratory activity: an interpretation of rats' behaviour in the open field and a light-dark preference test. Behav. Processes. 1, 153-164.

4. Bedse, G., Bluett, R. J., Patrick, T. A. (2018) Therapeutic endocannabinoid augmentation for mood and anxiety disorders: comparative profiling of FAAH, MAGL and dual inhibitors. Transl. Psychiatry. $8,92$.

5. Belzung, C., Pineau, N., Beuzen, A., Misslin, R. (1994) PD135158, a CCKB antagonist, reduces "state", but not "trait" anxiety in mice. Pharmacol. Biochem. Behav. 49, 433- 436.

6. Bourin, M., Hascoët, M. (2003) The mouse light/dark box test. Eur. J. Pharmacol. 463, 55-65.

7. Cancela, L. M., Bregonzio, C., Molina, V. A. (1995) Anxiolytic-like effect induced by chronic stress is reversed by naloxone pretreatment. Brain. Res. Bull. 36, 209-213.

8. Chaouloff, F., Durand, M., Mormède, P. (1997) Anxiety- and activity-related effects of diazepam and chlordiazepoxide in the rat light/dark and dark/light tests. Behav. Brain. Res. 85, 27-35.

9. Chen, Z., Wei, H1., Pertovaara, A., Wang, J., Carlson, S. (2018) Anxiety- and activity-related effects of paracetamol on healthy and neuropathic rats. Pharmacol Res Perspect. 6.

10. Costall, B., Domeney, A. M., Kelly, M. E., Tomkins, D. M., Naylor, R. J., Wong, E. H. F., Smith, W. L., Whiting, R. L., Eglen, R. (1993) The effect of the 5-HT3 receptor antagonist, RS 42358-197, in animal models of anxiety. Eur. J. Pharmacol. 234, 91-99.

11. Costall, Craig, K. J., Brown, K. J., Baum, A. (1995) Environmental factors in the etiology of anxiety. In: Bloom, F. E., Kupfer, D. J. (eds) Psychopharmacology: The Fourth Generation of Progress. Raven Press; New York, NY, 1325-1339.

12. Craig, K. J., Brown, K. J., Baum, A. (1995) Environmental factors in the etiology of anxiety. In: Bloom, F. E., Kupfer, D. J. (eds) Psychopharmacology: the Fourth Generation of Progress. Raven Press; New York, NY: 1325-1339.

13. Crawley, J., Goodwin, F. K. (1980) Preliminary report of a simple animal behavior model for the anxiolytic effects of benzodiazepines. Pharmacol. Biochem. Behav. 13, 167-170. 
14. Crawley, J. N. (1981) Neuropharmacologic specificity of a simple animal model for the behavioral actions of benzodiazepines. Pharmacol. Biochem. Behav. 15, 695-699.

15. Crawley, J. N. (1985) Exploratory behaviour models of anxiety in mice. Neurosci. Biobehav. Rev. 9 , $37-44$.

16. Crawley, J. N., Belknap, J. K., Collins, A., Crabbe, J. C., Frankel, W., Henderson, N., Hitzeman, R. J., Maxson, S. C., Miner, L. L., Silva, A. J., Wehner, J. M., Wynshaw-Boris, A., Paylor, R. (1997) Behavioural phenotypes of inbred mouse strains: implication and recommendations for molecular studies. Psychopharmacology 132, 107-124.

17. de Almeida Magalhães, T., Correia, D., de Carvalho, L. M., Damasceno, S., Brunialti Godard, A. L. (2017) Maternal separation affects expression of stress response genes and increases vulnerability to ethanol consumption. Brain Behav. 8, e00841.

18. Denenberg, V. H. (1969) Open-field behavior in the rat: what does it mean? Ann. N. Y. Acad. Sci. 159, 852-859.

19. Diaz, M. R., Mooney, S. M., Varlinskaya, E. I. (2016) Acute prenatal exposure to ethanol on gestational day 12 elicits opposing deficits in social behaviors and anxiety-like behaviors in Sprague Dawley rats. Behav Brain ResSep. 1, 310, 11-19.

20. Ennaceur, A., Chazot, P. L. (2016) Preclinical animal anxiety research - flaws and prejudices. Pharmacol. Res. Perspect. 4, e00223.

21. Fajemiroye, J. O., Adam, K., Jordan, K. Z., Alves, C. E., Aderoju, A. A. (2018) Evaluation of Anxiolytic and Antidepressant-like Activity of Aqueous Leaf Extract of Nymphaea Lotus Linn. in Mice. Iran J Pharm Res. 17, 613-626.

22. Fu, K., Miyamoto, Y., Sumi, K. (2017) Overexpression of transmembrane protein 168 in the mouse nucleus accumbens induces anxiety and sensorimotor gating deficit. PLoS One, 12, e0189006.

23. Gao, B., Cutler, M. G. (1992) Effect of acute administration of the 5-HT3 receptor antagonist, BRL $46470 \mathrm{~A}$, on the behavior of mice in a two compartment light-dark box and during social interactions in their home cage and an unfamiliar neutral cage. Neuropharmacology 31, 743-748.

24. Garcia, A. M. B., Martinez, R. C. R., Morato, S. (2008) Preference for the light compartment of a light/dark cage does not affect rat exploratory behavior in the elevated plus-maze. Psychol. Neurosci. 1, 73-80.

25. Hall, C., Ballachey, E. L. (1932) A study of the rat's behavior in a field. A contribution to method in comparative psychology. University of California Publications in Psychology 6, 1-12.

26. Haller, J., Alicki, M. (2012) Current animal models of anxiety, anxiety disorders, and anxiolytic drugs. Curr. Opin. Psychiatry 25, 59-64.

27. Haller, J., Aliczki, M., Gyimesine Pelczer, K. (2013) Classical and novel approaches to the preclinical testing of anxiolytics: a critical evaluation. Neurosci. Biobehav. Rev. 37, 2318-2330.

28. Hascoet, M., Bourin, M., Colombel, M. C., Fiocco, A. J., Baker, G. B. (2000) Anxiolytic like effects of antidepressants after acute administration in a four plate test in mice. Pharmacol. Biochem. Behav. $65,339-344$.

29. Jin, S., Zhao, Y., Jiang, Y. (2018) Anxiety-like behaviour assessments of adolescent rats after repeated maternal separation during early life. Neuroreport 29, 643-649.

30. Kliethermes, C. L. (2005) Anxiety-like behaviors following chronic ethanol exposure. Neurosci. Biobehav. Rev. 28, 837-850.

31. Kumar, R. S., Narayanan, S. N., Nayak, S. (2009) Ascorbic acid protects against restraint stressinduced memory deficits in Wistar rats. Clinics (Sao Paulo). 64, 1211-1217.

32. Laureano-Melo, R., da Silveira, A. L., de Azevedo Cruz Seara, F., da Conceição, R. R., da SilvaAlmeida, C., Marinho, B. G., da Rocha, F. F., Reis, L. C., Côrtes W da, S. (2016) Behavioral profile assessment in offspring of Swiss mice treated during pregnancy and lactation with caffeine. Metab. Brain. Dis. 31, 1071-1080.

33. Lukkes, J. L., Watt, M. J., Lowry, C. A., Forster, G. L. (2009) Consequences of Post-Weaning Social Isolation on Anxiety Behavior and Related Neural Circuits in Rodents. Front. Behav. Neurosci. 3, 18 
34. Mcllwain, K. L., Merriweather, M. Y., Yuva-Paylor, L. A., Paylor, R. (2001) The use of behavioral test batteries: effects of training history. Physiol. Behav. 73, 705-717.

35. Montgomery, K. C. (1958) The relation between fear induced by novel stimulation and exploratory behavior. J. Comp. Physiol. Psychol. 48, 254-260.

36. Narayanan, S. N., Kumar, R. S., Karun, K. M., Nayak, S. B., Bhat, P. G. (2015) Possible cause for altered spatial cognition of prepubescent rats exposed to chronic radiofrequency electromagnetic radiation. Metab Brain Dis. 30, 1193-1206.

37. Narayanan, S. N., Kumar, R. S., Paval, J., Kedage, V., Bhat, M. S., Nayak, S., Bhat, P. G. (2013) Analysis of emotionality and locomotion in radio-frequency electromagnetic radiation exposed rats. Neurol Sci. 34, 1117-1124. Epub 2012 Sep 14

38. Narayanan, S. N., Kumar, R. S., Paval, J., Nayak, S. (2010) Effect of ascorbic acid on the monosodium glutamate-induced neurobehavioral changes in periadolescent rats. Bratisl. Lek. Listy 111, 247-252.

39. Patro, G., Kumar Bhattamisra, S., Kumar Mohanty, B. (2016) Effects of Mimosa pudica L. leaves extract on anxiety, depression and memory. Avicenna J. Phytomed. 6, 696-710.

40. Ramos, A., Pereira, E., Martins, G. C., Wehrmeister, T. D., Izídio, G. S. (2008) Integrating the open field, elevated plus maze and light/dark box to assess different types of emotional behaviors in one single trial. Behav. Brain. Res. 193, 277-288.

41. Saitoh, A., Makino, Y., Hashimoto, T., Yamada, M., Gotoh, L., Sugiyama, A., Ohashi, M., Tsukagoshi, M., Oka, J., Yamada, M. (2015) The voltage-gated sodium channel activator veratrine induces anxiogenic-like behaviors in rats. Behav. Brain. Res. 292, 316-322.

42. Sarwar, R., Farooq, U., Naz, S. (2018) Isolation and Characterization of Two New Secondary Metabolites From Quercus incana and Their Antidepressant- and Anxiolytic-Like Potential. Front Pharmacol. 9, 298.

43. Scheinert, R. B., Haeri, M. H1, Lehmann, M. L., Herkenham, M. (2016) Therapeutic effects of stressprogrammed lymphocytes transferred to chronically stressed mice. Prog Neuropsychopharmacol. Biol. Psychiatry 70, 1-7.

44. Shastry, R., Ullal, S. D., Karkala, S., Rai, S., Gadgade, A. (2016) Anxiolytic activity of aqueous extract of Camellia sinensis in rats. Indian J. Pharmacol. 48, 681-686.

45. Shimada, T., Matsumoto, K., Osanai, M., Matsuda, H., Terasawa, K., Watanabe, H. (1995) The modified light/dark transition test in mice: evaluation of classic and putative anxiolytic and anxiogenic drugs. Gen. Pharmacol. 26, 205-210.

46. Sidor, M. M., Rilett, K., Foster, J. A. (2010) Validation of an automated system for measuring anxietyrelated behaviours in the elevated plus maze. J. Neurosci. Methods 188, 7-13.

47. Steimer, T. (2002) The biology of fear- and anxiety-related behaviors. Dialogues. Clin. Neurosci. 4, 231-249.

48. Takao, K., Miyakawa, T. (2006) Light/dark transition test for mice. J. Vis. Exp. 13, 104.

49. Young, R., Johnson, D. N. (1991) A fully automated light/dark apparatus useful for comparing anxiolytic agents. Pharmacol. Biochem. Behav. 40, 739-743.

50. Yu, C., Mei, X. T., Zheng, Y. P., Xu, D. H. (2015) Taurine zinc solid dispersions protect against coldrestraint stress-induced gastric ulceration by upregulating HSP70 and exerting an anxiolytic effect. Eur. J. Pharmacol. 762, 63-71. 